\title{
Inflammatory Fibroid Polyp causing small bowel intussusception: A case report
}

\author{
Iolanda Espirito Santo*†, Anne-Christine Cottier ${ }^{\dagger}$, Raffaella Sguinzi and Ian Fournier \\ Department of Visceral Surgery, Hospital of Sion, Av. Grand-Champsec 80, 1951 Sion, Switzerland \\ ${ }^{\dagger}$ Both authors contributed equally
}

\begin{abstract}
Inflammatory fibroid polyps (IFP), or Vanek's tumours, are rare lesions of the gastrointestinal tract whose clinical presentation and management vary according to lesion's size and location. We hereby present a case of an ileoileal intussusception accurately diagnosed by computer tomography. Laparotomy and small bowel resection were performed. A PDGFRA+ IFP was identified as the lead point and its rare CD117(c-kit) positivity emphasizes the histological variety of these lesions as well as the complexity thus inherent to a conclusive diagnosis.
\end{abstract}

\section{Introduction}

Adult intussusceptions are rare and account for approximately $5 \%$ to $10 \%$ of all intussusceptions, and only $1 \%$ of all intestinal obstructions [1]. Contrarily to children, in which $90 \%$ of cases of intussusception are idiopathic, $70 \%$ to $90 \%$ of cases in adults are secondary to a tumour or tumour-like lesion acting as a lead point that may be benign or malignant in nature. Etiologic entity and their clinical presentation are vast and distinct according to the lesion's location [2,3]. Inflammatory Fibroid Polyp (IFP) are one of such lesions, a rare idiopathic tumourlike and benign lesion of the gastrointestinal tract which, when located in the small bowel, can act as lead point for an intussusception. A consensus is yet to be found regarding preoperative diagnosis, but clinical management requires explorative laparotomy [3]. We hereby present a case of an adult ileoileal intussusception, diagnosticated preoperatively, managed by open surgery and subsequently attributed to an Inflammatory Fibroid Polyp. Even though it is not difficult to find reports of such lesions in literature [3,5,9], our particular histological findings of a CD34+CD117+ lesion, raises awareness to the existence of different hystological variants and antigen expressions of the IFPs and emphasizes the complexity of their diagnosis [4-6].

\section{Case presentation}

A 60-year-old healthy female, presented in the emergency department with a 3-day history of diffuse abdominal pain associated with nausea and vomiting with no hematemesis. The patient also suffered from absence of stools for the 3 days prior to her admission. There was no history of rectal bleeding. She reported a weight loss of two kg since the beginning of the described symptoms. Upon physical examination, her vital signs were stable. Abdominal examination revealed mild distention, tenderness and pain in all quadrants. There were no signs of acute peritonitis and no palpable mass. We did not hear high-pitched bowel sounds. Biochemical investigations were followed at the emergency station and showed a mild inflammatory syndrome. The white blood cell (WBC) was $11 \mathrm{G} / \mathrm{L}$ (normal: $4-10 \mathrm{G} / \mathrm{L}$ ) and C-reactive protein was $5.6 \mathrm{mg} / \mathrm{L}$ (normal $<5 \mathrm{mg} / \mathrm{L}$ ). Other blood tests were normal.
Bowel obstruction was clinically suspected, and a contrast enhanced abdominal scan with contrast enema was performed and revealed a mechanical ileus with up to $50 \mathrm{~mm}$ in diameter dilatation of the small intestines. The finding of a target-mass sign confirmed that an intussusception of two adjacent ileal segments was he likely cause of the ileus (Figure 1). There were indirect signs of intestinal distress, like free fluid in the lower intestinal quadrants. No lead point for the ileus was clearly identified on imaging.

A nasogastric tube was placed, and the patient underwent a laparotomy. The small bowel exploration confirmed an intussusception adjacent to palpable intraluminal mass. With limited manipulation of this segment, a $36 \mathrm{~cm}$ length segmental resection of the small bowel, 50 $\mathrm{cm}$ from the jejuno-ileal junction with side-to-side anastomosis and lymph node dissection was performed.

On full exploration of the abdomen, there is no carcinosis on the colon. Postoperative outcomes were good and without complications. The patient was discharged 4-days following the surgical procedure.

The surgical specimen revealed the diagnosis of inflammatory fibroid polyp. The ileal surgical specimen contained a polypoid mass measuring $4.9 \times 3 \times 2.8 \mathrm{~cm}$ (Figure 2) which was sent to histological examination. An abundant eosinophil and mastocyst CD117+ inflammatory infiltration were found. The results showed weak positivity for CD34+ and smooth muscle actin. DOG1, Desmine and S100 were negative. The lesion, located $12 \mathrm{~cm}$ from the proximal section and more than $10 \mathrm{~cm}$ from the distal section, was centred in the submucosa and ulcerated on the surface. The muscular mucosa and sub serosa did not show any particularities. There were no signs of malignancies. The lymph nodes did not reveal any metastasis. The platelet derived growth factor receptor alpha (PDGFRA) mutation was detected.

*Correspondence to: Iolanda Nazaré da Graça do Espírito Santo, Route de la Feuillère 13, 1010 Lausanne Switzerland, Tel: +41797840758; E-mail: iolandaespiritosanto@gmail.com

Key words: inflammatory fibroid polyp, IFP, intussusception, bowel, GIST

Received: April 08, 2021; Accepted: April 18, 2021; Published: April 24, 2021 


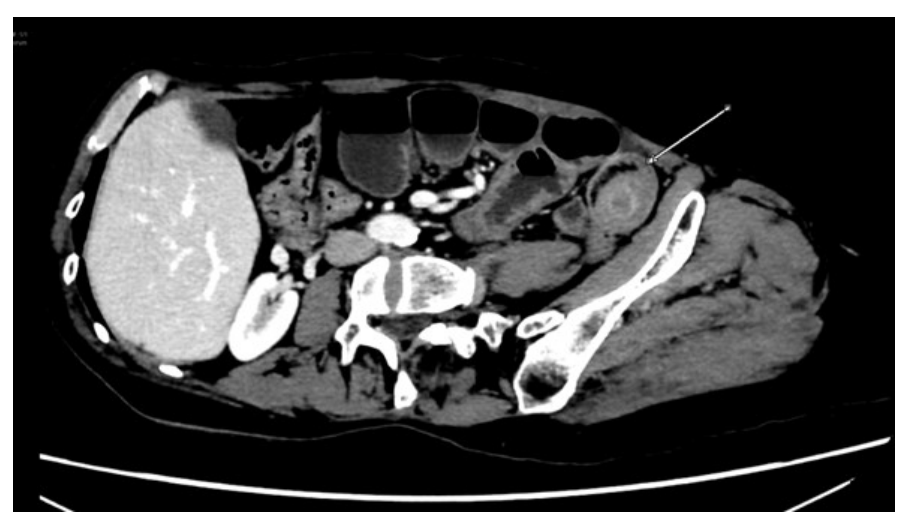

Figure 1. Axial abdominal computer tomography revealing a bowel intussusception's target-mass image (arrow) associated to a mechanical ileus

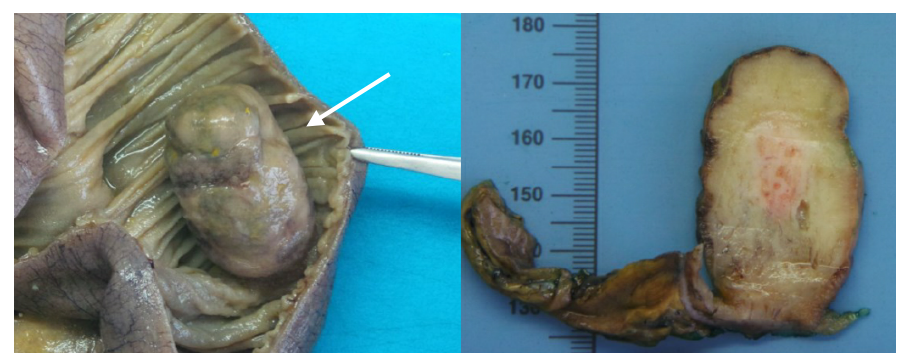

Figure 2. Postoperative specimen revealing an intraluminal polypoid mass (arrow) measuring $4.9 \times 3 \times 2.8 \mathrm{~cm}$

\section{Discussion}

Described since 1674, intussusception in one of rare cases of intestinal obstruction- a common surgical emergency $[7,8]$ [Barbette, 1674; Johan, 2020]. Intussusception is defined as the invagination of a proximal portion of the bowel along with its mesentery into a more distal one, due to the presence of a intraluminal lesion or irritant also known as lead point -, altering normal bowel peristalsis $[9,10]$. According to the segment involved, intussusception may be classified as ileoileal, ileocolic, colocolic and colorectal. Small bowel lead points are mostly benign in nature (lipomas, hamartomous polyps, inflammatory polyps, hyperplastic polyps and Meckel's diverticulum, Peutz-Jager Syndrome, Henoch Schoenlein Purpura and tuberculosis), although there have been reports of metastatic lesions. Contrarily, large bowel lesions are frequently associated to a malignant etiology $[2,4,6,7]$. This discrepancy accounts for the different management approaches proposed and described ahead.

In our report, an IFP was the cause of an ileoileal intussusception. Recent studies have shown that these lesions are rare and mostly present in around the fifth to seventh decades $[3,6]$. Even though a slight female predominance has been reported, the female-to-male ratio may vary from ranging from 2.8: 1 to $0.7: 1$. [5,6] IFPs were first described by Vanek as a gastric submucosal granuloma with eosinophilic infiltration [12], and subsequently identified throughout the gastrointestinal tract, having earned the term IFP proposed by Helwig ad Rainier in 1953 [13]. These mesenchymal tumours are indeed submucosal in origin and consist of fibroblast-like spindle cells as inflammatory infiltrates (eosinophiles, mast cells) arranged concentrically around blood vessels ("onion skin"). Most masses range form $2-5 \mathrm{~cm}$ but lesions up to $20 \mathrm{~cm}$ have been reported $[14,15]$.

IFPs mostly go unnoticed and typically are incidental findings during endoscopy or laparoscopy or laparotomy procedures [3]. They are mostly found in the gastric antrum (66-75\%), followed by the small bowel (18-20\%), colorectal region (4-7\%), gallbladder (1\%), oesophagus $(1 \%)$, duodenum $(1 \%)$ and appendix $(>1 \%)$. Clinical presentation can be acute, subacute or chronic and depends on tumour location and size. Gastric lesions usually present with epigastric pain and bleeding, whereas pain obstruction due to intussussepcion - as occurred in our report- are the most the frequent presentation in the small bowel $(17.0 \%-77 \%, 27 \%-12.5 \%$, respectively) $[6,16]$. Other gastrointestinal symptoms include vomiting, diarrhoea, tenesmus, alterations in bowel habits, and weight loss. Gastrointestinal bleeding, though less frequent in the small bowel lesions, may suggest mucosal ulceration and/or bowel ischemia, especially in case of an intussusception $[3,17,18]$.

Preoperative diagnosis includes blood evaluation and radiological imaging. The typical radiological sign is a target-mass or sausage-shape mass with layering effect as a result of a longitudinal compression and venous congestion of the intussusceptum [8,9]. Abdominal CT scans have become the standard of care, with a sensitivity between 50 and $100 \%$, an accuracy of $58-100 \%$ and a specificity of $57 \%$ to $71 \%$ in the diagnosis of intussusceptions. The higher values in these ranges are related with more recent reports with the increase in CT resolution $[10,16,17]$.

Imaging plays a decisive role in the diagnosis of IFPs. In case of intussusception in adults, CT scan is favored over ultrasound [10]. With a sensitivity between 50 and $100 \%$, the preferred diagnostic imaging technique today is the abdominal CT scan. [Haley et Brian]. Note that colonoscopy can be useful in cases of intussusception of the colon. Well-defined, round or ovoid endoluminal masses hyperenhanced adjacent mucosa on CT scan are features usually visualized [10]. However, it is important to note that it is rare to visualize a mass on imaging in cases of intussusception [19]. The limited use of CT may be due to the emergency situation of patients needed a potential surgical treatment. Magnetic resonance imaging is not commonly required for the diagnosis of adults or children intussusception [20].

The role of colonoscopy consists in the reduction of the invagination and thus the resolution of th2 mechanical emergency. Biopsy or polypectomy remain under debate because of the risk of bleeding al also spreading of tumour cells. Intussusception remains a surgical emergency, because of the risk for bowel ischemia, necrosis and subsequently perforation. Laparotomy is considered standard of care, but recent reports have advocated for the use of laparoscopy specially when preoperative diagnosis is unclear [2,8-10,21,22].

A consensus is also yet to be found regarding the extent of the surgical resection, as some authors suggest en bloc resection of the intussuscepted segment while others prefer its initial reduction allowing for a targeted, more selective resection. The en bloc approach is broadly implemented in large bowel intussusceptions and frequently associated with oncologic resection with lymphatic curage due to the higher probability of malignancy. On the other hand, the mostly benign lesions of the small bowel have been seeing a rise in the reduction-resection technique $[9,10]$. We decided to implement an en bloc resection in our patient having in mind the - even if small-, non-negligible incidence of malignancy in small bowel intussusception ( 1 - 47\% ileoileal vs 43 $-100 \%$ in ileocolic and colocolic intussusceptions) and the inherent inability to accurately differentiate benign form malignant etiologies intraoperatively. Furthermore, IFP resection is usually curative since there have been no reports of recurrence in literature [6].

To this time, the aetiopathogenesis of IFPs remains elusive, but they have been considered reactive non-neoplastic lesions, to whom 
several inciting events have been attributed such as trauma, allergic reaction, bacterial, chemical, physical stimuli [3]. More recently data has supported the presence of a genetic tendency in connection with the expression and activation of platelet-derived growth factor receptor alpha (PDGFRA) mutations in up $90 \%$ of cases [23]. Reports have confirmed a sustained expression of PDGFRA and activation of it mutations by IFPs, thus, suggesting of a potentially neoplastic pathogeny. Similar mutations in this gene have been found in several tumours, namely peripheral nerve sheath tumours, glioblastomas and gastrointestinal stromal tumours (GIST), which suggest a common oncogenetic pathway $[14,19]$. Differential diagnosis between GIST and IFP may be difficult, since both predominantly occur in the stomach, as a polypoid mass and since a small bowel GIST may also present as an intussusception. IFPS, contrary to GIST, may arise in any segment of the gastrointestinal tract. As their size increases, IFPs may present different histological patterns: classical, nodular, sclerotic and edematous, or a combination thereof $[6,24]$. Nonetheless, they remain histologically different from GIST, since IFP's spindle cells have small-flattened nuclei and GIST have epithelioid or mixed phenotype with oval ones. Furthermore, it has been suggested that immunohistochemistry would accurately distinguish GIST from IFPs, since both are CD34+, but only GIST are also CD117(c-kit)+ $[3,14]$. Even so, there have been seldom reports of CD117+, thus suggesting inherent diagnostical difficulties that may be related to the different histological varieties of IFP [4-6].

\section{Conclusion}

Intussusceptions are rare, potentially severe clinical entities that must not go unrecognized. Several tumour or tumour-like lesions may be involved, rarely, an IFP. Clinical and radiological presentation and anatomical location are of utmost importance to guide surgical approach. Nevertheless, these findings are frequently equivocal, rendering histopathological features crucial to the diagnosis.

\section{Declarations}

Financial Support and Sponsorship The authors have no relevant financial or non-financial interests to disclose.

\section{Conflicts of interest}

The authors have no conflict of interest to declare.

\section{Acknowledgements}

None.

\section{References}

1. Barussaud M, Regenet N, Briennon X (2006) Clinical spectrum and surgical approach of adult intussusceptions: A multicentric study. Int J Colorectal Dis 21: 834-839. [Crossref]

2. Shimazaki J, Nakachi T, Tabuchi T, Suzuki S, Ubukata H (2015) Laparoscopic management of an octogenarian adult intussusception caused by an ileal lipoma suspected preoperatively: a case report. World J Surg Oncol 22: 13-75.
3. Akbulut S (2012) Intussusception due to inflammatory fibroid polyp: a case report and comprehensive literature review. World J Gastroenterol 18: 5745-52. [Crossref]

4. Makhlouf HR, Sobin LH (2002) Inflammatory myofibroblastic tumors (inflammatory pseudotumors) of the gastrointestinal tract: How closely are they related to inflammatory fibroid polyps? Hum Pathol 33: 307-315.

5. Wysocki AP, Taylor G, Windsor JA (2007) Inflammatory fibroid polyps of the duodenum: A review of the literature. Dig Surg 24: 162-168.

6. Garmpis N, Damaskos C, Garmpi A (2021) Inflammatory fibroid polyp of the gastrointestinal tract: A systematic review for a benign tumor. In Vivo (Brooklyn) 35: 81-93.

7. de Moulin D, Paul Barbette (1985) A seventeenth-century Amsterdam author of bestselling textbooks. Bull Hist Med 59: 506-514.

8. Johan S, Lee PP, Azizan N, Hayati F, Zakaria AD (2020) Vanek's tumor causing ileoileal intussusception in a middle-aged man. Oxford Med Case Reports 2020.

9. Yakan S, Calıskan C, Makay O, Deneclı AG, Korkut MA (2009) Intussusception in adults: Clinical characteristics, diagnosis and operative strategies. World $J$ Gastroenterol 15 .

10. Al Taei TH, Al Mail SA (2018) Small bowel intussusception due to inflammatory fibroid polyp: A case report. Radiol Case Reports 13: 801-804.

11. Kang SH, Kim SW, Moon HS, Sung JK, Jeong HY (2015) Coloproctology Inflammatory Fibroid Polyp in the Jejunum Causing Small Bowel Intussusception. Ann Coloproctol 31: 106-109.

12. VANEK J (1949) Gastric submucosal granuloma with eosinophilic infiltration. Am J Pathol 25: 397-411.

13. Helwig EB, Ranier A (1953) Inflammatory fibroid polyps of the stomach. Surg Gynecol Obstet 96: 335-367.

14. Schildhaus H, Cavlar T, Binot E, Wardelmann E (2008) Inflammatory fibroid polyps harbour mutations in the platelet-derived growth factor receptor alpha (PDGFRA) gene. J Pathol 216: 176-182.

15. Mohamud SO, Motorwala SA, Daniel AMR, Tworek JA, Shehab TM (2008) Giant ileal inflammatory fibroid polyp causing small bowel obstruction : a case report and review of the literature. Cases $J 5: 1-5$.

16. Amr MA, Polites SF, Alzghari M, Onkendi EO, Grotz T (2015) Intussusception in adults and the role of evolving computed tomography technology. Am J Surg 209: 580-583. [Crossref]

17. Maghrebi H, Makni A, Rhaiem R (2017) Adult intussusceptions: Clinical presentation, diagnosis and therapeutic management. Int J Surg Case Rep 33: 163-166.

18. Report C, Sözen S, Uzun AS, Erdem H, Daş K (2013) Ileoileal Intussusception. European Journal of General Medicin 10: 236-238.

19. Adams HS, Bergstrom B, Haines B, Roberts N (2017) Inflammatory Fibroid Polyp: An Unusual Cause of Ileoileal Intussusception. Case Rep Surg 2017: 6315934. [Crossref]

20. Ciftci F (2015) Diagnosis and treatment of intestinal intussusception in adults: A rare experience for surgeons. Int J Clin Exp Med 8: 10001-10005.

21. Kang S Il, Kang J, Kim MJ (2014) Case Report Laparoscopic-Assisted Resection of Jejunojejunal Intussusception Caused by a Juvenile Polyp in an Adult. Case Rep Surg 2014: 856765 .

22. Siow SL, Mahendran HA (2014) O RIGINAL A RTICLE A Case Series of Adult Intussusception Managed Laparoscopically. Surg Laparosc Endosc Percutan Tech 24: 327-331. [Crossref]

23. Pinto-Pais T, Fernandes S, Proença L (2015) A large gastric inflammatory fibroid polyp. GE Port J Gastroenterol 22: 61-64.

24. Kim YIL, Kim WH (1988) Inflammatory fibroid polyps of gastrointestinal tract: Evolution of histologic patterns. Am J Clin Pathol 89: 721-727.

Copyright: (C2021 Santo IE. This is an open-access article distributed under the terms of the Creative Commons Attribution License, which permits unrestricted use, distribution, and reproduction in any medium, provided the original author and source are credited. 